\title{
A Visual Analytics Approach for Assessing Pedestrian Friendliness of Urban Environments
}

\author{
Tobias Schreck, Itzhak Omer, Peter Bak and Yoav Lerman
}

\begin{abstract}
The availability of efficient transportation facilities is vital to the function and development of modern cities. Promoting walking is crucial for supporting livable communities and cities. Assessing the quality of pedestrian facilities and constructing appropriate pedestrian walking facilities are important tasks in public city planning. Additionally, walking facilities in a community affect commercial activities including private investment decisions such as those of retailers. However, analyzing what we call pedestrian friendliness in an urban environment involves multiple data perspectives, such as street networks, land use, and other multivariate observation measurements, and consequently poses significant challenges. In this study, we investigate the effect of urban environment properties on pedestrian movement in different locations in the metropolitan region of Tel Aviv. The first urban area we investigated was the inner city of the Tel Aviv metropolitan region, one of the central regions in Tel Aviv, a city that serves many non-local residents. For simplicity, we refer to this area as Tel Aviv. We also investigated Bat Yam, a small city, whose residents use many of the services of Tel Aviv. We apply an improved tool for visual analysis of the correlation between multiple independent and one dependent variable in geographical context. We use the tool to investigate the effect of functional and topological properties on the volume of pedestrian movement. The results of our study indicate that these two
\end{abstract}

\author{
T. Schreck ( $\square)$ \\ University of Konstanz, Konstanz, Germany \\ e-mail: tobias.schreck@uni-konstanz.de \\ I. Omer · Y. Lerman \\ Tel Aviv University, Tel Aviv, Israel \\ e-mail: omery@post.tau.ac.il \\ Y. Lerman \\ e-mail: yoavlerm@post.tau.ac.il \\ P. Bak \\ IBM Research Lab, Haifa, Israel \\ e-mail: peter.bak@il.ibm.com
}


urban areas differ greatly. The urban area of Tel Aviv has much more correspondence and interdependency among the functional and topological properties of the urban environment that might influence pedestrian movement. We also found that the pedestrian movements as well as the related urban environment properties in this region are distributed geographically in a more equal and organized form.

\section{Introduction}

Past attempts to explain pedestrian or walking movement patterns in urban environments mainly focused on two groups of factors that affect this movement: street connectivity and functional factors. Previous studies have applied multivariate and bivariate regression models, mostly by the Stepwise method, to obtain the highest coefficient of determination, by using the R2 measure for example [such as (Hillier et al. 1993) or (Ozbil et al. 2011)]. However, we know little about how the spatial structure of street networks and street connectivity, i.e. topological centrality, interacts with other important urban environment functional factors such as commercial land use and sidewalks in different urban environments, and what are the consequences of that interaction concerning pedestrian movement. Motivated by these questions, we investigated the correlation of urban environment properties on pedestrian movement, as measured by the number of pedestrians per time in different urban areas in the metropolitan region of Tel Aviv. Specifically, we investigated the inner city of the metropolitan Tel Aviv, a city that serves many non-local residents. We also investigated Bat Yam, a smaller city that serves mostly local residents. Our key research question asks for the spatial-functional configurations that enhance walkability (or pedestrian friendliness) in these urban environments. While many qualitative factors contribute to the walkability or pedestrian friendliness, in this work we assume the number of pedestrians is an indicator therefore, recognizing this is a simplification. We want to explore how these configurations create pedestrian friendly environments. The results of this study can potentially guide urban development policy in assigning priority to some of the identified properties.

The analytic problem in our case is a correlation problem, involving a set of four independent variables that describe the spatial and functional properties and one dependent variable-namely, the average number of pedestrians. Consequently, we need to also consider the geospatial map and the overall street network in the analysis. To address this challenging problem, we rely on approaches from visual data analysis to make sense of the acquired data. Specifically, we applied a method for visual cluster analysis that groups the sets of observations into a smaller number of categories, describing similar configurations of the independent variables. Using this, along with a linked map display, we then analyzed the dependency of the variables in terms of attributes and geospatial position in the 
map context. We thereby provided both an analytical workflow to a general problem but also practical insights for the two specific studied cities, enabling us to give answers to questions of critical importance for urban planning.

The remainder of this chapter is structured as follows. In Sect. 2, we describe related work. In Sect. 3, we introduce the geospatial analysis problem at hand and describe our data acquisition process. In Sect. 4, we describe the setup of the visual analysis tool we implemented. In Sect. 5, we explain how we applied our tool on the collected data, discussing the main insights found and their implications. Finally, in Sect. 6, we summarize our approach along with interesting future work in the area.

\section{Related Work}

In this section, we review related work that analyzed data on urban environments with a focus on pedestrian movement patterns. We also briefly introduce related work on the visual analysis of data in geospatial and multivariate domains. Visual and analytical comparison between spatial distributions of objects and attributes within a GIS framework can be an essential tool for understanding and explaining geographic phenomena in urban areas.

Much evidence has been collected indicating that the geographic distribution of pedestrian movement along city streets is affected by two main characteristics of the urban environment - topological centralities of streets (or street segments) and the spatial distribution of retail and service facilities [e.g., (Golledge and Stimson 1997; Hillier et al. 1993; Jiang 2007; Ozbil et al. 2011)]. However, we still have no sufficient knowledge on these relationships, i.e., why, when, and where certain urban environment characteristics are more influential than others for predicting pedestrian movement in the city. This complexity may be related to the fact that the topological properties of individual streets are significantly correlated to the spatial distribution of retail and services [e.g., (Desyllas et al. 2003; Porta et al. 2006)]. Many studies were conducted to address this issue by combining various attributes in empiric investigations of urban pedestrian movement [e.g., (Orellana and Wachowicz 2011; Ozer and Kubat 2007; Raford and Ragland 2006; Torrens 2012)]. In practice, however, and as mentioned above, most investigations of the relationship among pedestrian movement, street network connectivity, and land use distribution have been conducted in statistical terms only, with no explicit and detailed consideration of geographic and multidimensional aspects.

Visual and analytical comparisons between spatial distributions of objects and attributes within a GIS framework can be an essential tool for understanding and explaining geographic phenomena in urban areas. They can do so, by offering an integrated view among multiple dimensions, including independent and dependent quantitative variables, in a geographic context. Accordingly, our approach to analyze pedestrian friendliness data is based on visual representations that compare and correlate the data from these perspectives. The recently evolving field of visual 
analytics addresses the design, application and evaluation of tools that combine automatic data analysis methods with visual-interactive representations (Keim et al. 2011; Thomas and Cook 2005). Recently, visual-interactive approaches have also been extensively applied in the geographic data analysis community (Andrienko and Andrienko 2006). For understanding multivariate data, projection or clustering methods are often applied to group data by similarity and thereby ease their interpretation. Techniques for such grouping include principal components analysis as a prominent dimensionality reduction technique (Jolliffe 2002), and the selforganizing maps algorithm for projection and cluster analysis (Bak et al. 2010; Guo et al. 2005; Spielman and Thill 2008; Kohonen 2001).

\section{Data Acquisition for Pedestrian Friendliness Analysis}

Trends show that cities are becoming more populated, and the analysis and improvement of city infrastructure is an important goal. We acquired empirical data for a study to identify the influential factors that can determine the pedestrian friendliness of a city street network. To this end, we measure a set of independent variables that may explain the attractiveness of street properties to pedestrians, useful for city analysis and policy planning. In this section, we describe the setup of the data acquisition, which is the basis for the subsequent analysis.

\subsection{Tel Aviv and Bat Yam: Background}

We present research that deals with pedestrian movement in two urban areas- the cities of Tel Aviv and Bat Yam, focusing on the effect of the street network structure and land uses on the intensity of pedestrian movement. Both areas include orthogonal street patterns (similar to a grid). Yet, they also show hierarchical patterns as well as many internal loops, cul-de-sacs, and $\mathrm{T}$ junctions that characterize more modern planning approaches. The selected study area in Tel Aviv is divided into two sections that were designed and built at different times and have different characteristics. The western section was built during the 1930s according to a master plan made by the Scottish urban planner, Sir Patrick Geddes. The eastern region was built during the 1950s as part of the "East Tel Aviv" plan. These two areas differ in residential density, street grid, and land use mix. The city of Bat Yam has 130,000 residents. The study area in Bat Yam included the entire city, which has parts that were built based on plans made in the 1930s in the northwest area of the city, and parts that were built later in the 1950s and 1970s, in the south and to the east. The newer parts of the city have street networks that are less connected than the older parts. 


\subsection{Data Acquisition}

Measurement points in the study areas in Tel-Aviv and Bat-Yam were selected to represent a range of different centrality measures and distribution of land uses. In both cities data was collected using the gate count method where each pedestrian who passed through the gate was counted. This method has been used by other studies (Desyllas and Duxbury 2000; Zhang et al. 2012) providing high-resolution count results efficiently for such as those that were conducted in this research. The selected research area in Tel Aviv covered 400 acres. Concerning its central location in the metropolitan region, we assume that non-local residents make up a significant portion of pedestrian movement. The count was done for 5 min every hour for $5 \mathrm{~h}$ at each survey point. The measurement took place on a sunny weekday between the hours 3 and 8 p.m. The survey took place in 51 different street segments at 95 measurement points. Bat-Yam is a suburb of Tel Aviv, therefore we assume that the pedestrians movements in this city are mainly due to local residents. The research area in Bat-Yam includes the entire city area, which spreads over 1,800 acres. Pedestrian volume sampling was done in 69 street segments (122 measurement points) throughout Bat Yam. At each survey point the count was done for 5 min every hour for $8 \mathrm{~h}$. The Bat Yam pedestrian survey was done on a weekday during the hours 7 a.m. until Noon and from 3 to 8 p.m. In both cities, we collected data to understand the correlations between the built environments and the pedestrian movement. Specifically, we collected data that would enable investigating the correlations of the street network properties and the land use properties with regard to pedestrian movements, in different parts of each area and on different geographical scales. Although the sampling took place during slightly different hours in both cities, the overall average distribution of pedestrian movement during the measured period shows similar patterns in both areas.

In the two areas we investigated, the data for the functional independent variables were partially collected using a field survey and partially by using geographic information layers. Data on the land-use distribution was obtained as GISlayers from the Survey of Israel (MAPI) and the Mapa company. ${ }^{1}$ The data include geo-referenced residential and public buildings and a detailed description of their land uses. The street connectivity independent variables-namely, connectivity and local integration-were measured at the level of individual street segments by using the space syntax methodology (Hillier 1996). When using this methodology, the built environments' spatial configuration is described by means of a topological analysis of its axial map. An axial map is defined as the smallest set of the longest lines of direct visibility and movement that pass through all of a citys' open spaces. For any particular axial line, connectivity denotes the number of directly linked axial lines. The integration measure indicates the closeness of an axial line to other axial lines by computing the shortest distance (or step depth) of

\footnotetext{
1 The Survey of Israel, the Israeli official government agency for Mapping, Geodesy, Cadastre and Geoinformatics. Mapa is a private company. All data is updated to 2011.
} 
the respective line from other axial lines in a given area. The local integration measure describes integration only up to a defined radius of topological distance (number of steps), which is restricted to three steps in the current research. To create the axial maps of the neighborhoods and the entire city as well as to compute the street connectivity independent variables, we used two software programs: Depthmap and AxialGen. Depthmap [(Turner 2004), version 8.15, UCL] was used to automatically create (and manually edit) axial maps based on the urban street network. AxialGen [(Jiang and Liu 2010), version 1.0] was used to calculate, analyze, and present the connectivity independent variables (space syntax attributes) within the ArcMap (ver. 9.3) GIS software. The data for the dependent variable were collected using a survey in selected street segments that represent geographical locations and street kinds in both of the studied areas.

\subsection{Attribute Formation and Data Summary}

In both Tel Aviv and Bat Yam, the street segments for the surveys were selected so that a range of topological and functional values would be represented in the sample. We compiled 122 measurement points for Bat Yam and 95 measurement points for Tel Aviv. As discussed above, at each measurement point the pedestrian movement was sampled during a few hours using the gate count method. The following list summarizes the four independent variables and the one dependent variable we used. This data was input to the visual analysis approach described in the next section.

- CommFront: Binary variable, indicating whether a retail commercial front is present at the measurement point (independent).

- BusStation: Binary variable, indicating whether a bus stop is present at the measurement point (independent).

- Connectivity: Axial connectivity value of the street network at the measurement point (independent).

- LocalInt: Axial local integration value of the street network at the measurement point (independent).

- AveragePerHour: Number of pedestrians counted at the measurement point, averaged per hour (dependent).

\section{Visual Analysis Design}

In this section, we describe the system design by its components. The design is derived from the main problems of analyzing the data at hand and is inspired by the workflow presented in (Bak et al. 2010): 
1. We form groups of locations that are similar regarding their topological and functional configurations.

2. We visually compare the configuration groups against one another in terms of the distribution of topological and functional attribute values.

3. Then, we correlate the configuration groups against the target variable (the average number of pedestrians).

4. We also analyze the spatial distribution of the configurations and pedestrian counts over the map, giving the geographic context of the observations.

We address the first step in the workflow (1) by applying the self organizing map algorithm (SOM) (Kohonen 2001) to the four-dimensional configuration data samples. The method is a neural-network type data reduction and projection algorithm, which is often used in visual cluster analysis. We use it to obtain from the larger number of configurations, a small number of representative (cluster) configurations, on which the subsequent analysis is based. Training a small SOM of size $3 \times 2$, we obtained four main distinct clusters (configurations) contained in the four corner fields of the $3 \times 2$ map. Note that the size of the SOM grid influences the number of prototypes one obtains. The SOM algorithm does not by itself yield an indication of the number of clusters one can assume in the data, but this needs to be done by inspection of the analysis. In our case, we tried also larger resolutions but found empirically, that a $3 \times 2$ grid yields four distinct configurations which are suitable to our task at hand. We also note that the two central fields in the SOM result remained unpopulated interpolation fields - an indication that the found prototypes are rather discriminative against each other.

The Visual Analytics approach in our design is reflected by enabling users to iteratively define the input parameters of the SOM algorithm (size of the grid, and consequently the number of obtained prototypes). This interaction allows the user to decide when the resulting prototypes are significant enough; that is, when the number of contained data instances is sufficiently large for each luster, and the prototypes are discriminative enough. In addition, Visual Analytics as a core component in our approach is expressed through the interactive linkage between the visualization elements, resulting in a feedback loop between results of the algorithm and the users' search for insight. In this context, visualization is used as the interface to algorithmic refinement and an interactive display of algorithmic results.

We visualize each configuration group by a radial parallel coordinate (or radar) chart glyph, on which the four dimensions span a planar coordinate system. The mapping, clock-wise from the top is: CommFront, BusStation, Connectivity, LocalInt. Figure 1 (left and middle) shows an illustration. A black polyline represents the average values of the cluster (the cluster prototype) in terms of these independent variables. Yellow semitransparent bands indicate the distribution of actual measurements represented by the cluster. We also color-code the average number of pedestrians observed at the respective configurations as the background color. There, light shades represent low pedestrian counts and dark shades represent high counts [colors used according to (Brewer 2012)]. 

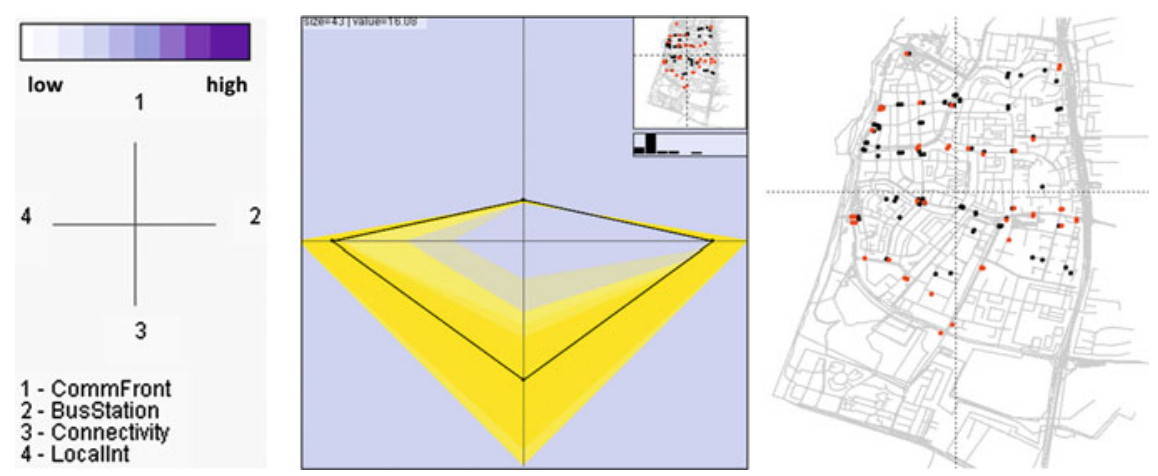

Fig. 1 The components of our visual analysis design. A four-dimensional glyph represents clusters of similar configurations of the input variables (left). The glyph represents the average number of pedestrians observed for a group of similar street configurations by color-coding the back-ground and by a histogram (darker indicates larger numbers). A dot map display shows the occurrence of measurements represented by this cluster on the map (right, highlighted by bright/ red dots)

We extend the glyph to include a small dot map inset which shows the geographic position of the represented observations. Also, we show the distribution of the pedestrian counts represented in the groups by a histogram [see Fig. 1 (middle, top-right)]. Our approach also allows for zooming in the dot map for a more detailed inspection of the spatial distribution of configurations.

The visualization decisions for the glyph construction are taken consciously and in close cooperation with domain experts. The four-dimensional glyph was chosen to represent the input variables using a radar-chart. Literature suggests (Inselberg 2002) the effectiveness and expressiveness of this representation to detect patterns in datasets with multidimensional attributes. The choice of using the radar-chart version of this representation type was to save real-estate on the display. As our users were exclusively with geographic background, their request for corresponding spatial representation was accommodated by the map view. The radar-chart view and the map-view were used in a highly integrated manner, in order to generate hypotheses and reflect on the distinctiveness of the SOM clusters. We are convinced that the existing design is a good starting point. Future work may include a systematic usability test which could be the basis for further improvements on the analytical workflow.

\section{Pedestrian Friendliness Analysis for Bat Yam and Tel Aviv}

Our main question is how do the spatial structures of street networks and street connectivity interact with other important functional factors such as commercial land use in their effects on pedestrian frequency in different urban environments in 
the same metropolitan region. That is, how do different spatial-functional configurations affect urban pedestrian movement in different geographical conditions? Based on visual data analysis, we address aspects of this question in the following.

\subsection{Bat Yam Analysis}

We first consider the Bat Yam case. We start our analysis by clustering the observation data according to the functional and topological measurements (see Sect. 4). The SOM analysis of the Bat Yam data reveals four distinct clusters of spatial-functional configurations (see Fig. 2, top row). In the radar chart, the four directions, up/east/south/west represent the independent variables CommFront, BusStation, Connectivity, and LocalInt (see also legend in Fig. 1 left). We can see at a glance that: (1) in general, the values of functional variables are strongly related to the values of the topological variables - the four-dimensional glyphs tend to expand rather equally in all directions, which means an interdependency among these variables is present. This is a side results of our analysis. And (2) a positive correlation exists between the values of the spatial-functional independent variables and the dependent data, as the frequency of pedestrian movements increases, represented by the background color in the image. The background color gets darker (more pedestrians) as the four-dimensional glyph shape becomes larger. This illustrates well the effect of the built environment's properties on pedestrian movement in the city.

Moreover, a detailed examination of the four configurations reveals that high spatial-functional values provide sufficient conditions for high volumes of pedestrians. The large cluster that represents walkable configurations in Fig. 2 (top row, left-most cluster) illustrates that well-showing higher values in all the independent values with small (standard) deviation. However, as the second cluster from the left in the row illustrates, high values of independent variables are not necessary conditions of pedestrian friendliness; lower values in one or in several variables still coincide with high volume of pedestrian movement. This is exemplified by the relatively lower values and higher deviations in the Connectivity and the BusStation variables, as apparent from the larger spread bands shown in yellow in the glyph. This means that high values of variables, mainly of local integration and commerce, are necessary for significant pedestrian movement. The other two configurations of variables that characterized low volume of pedestrian movement illustrate that the variable of commerce is low (with a small standard deviation) for both.

In light of the results described above, we found it necessary to examine the geographical patterns of the configurations that are identified in the SOM process and their locations in the urban environment. Such examinations are possible through maps that display the location of the members of each cluster. Figure 2 (middle row) displays the configuration locations represented by the respective clusters. We found that the street segments that are represented by the two most 

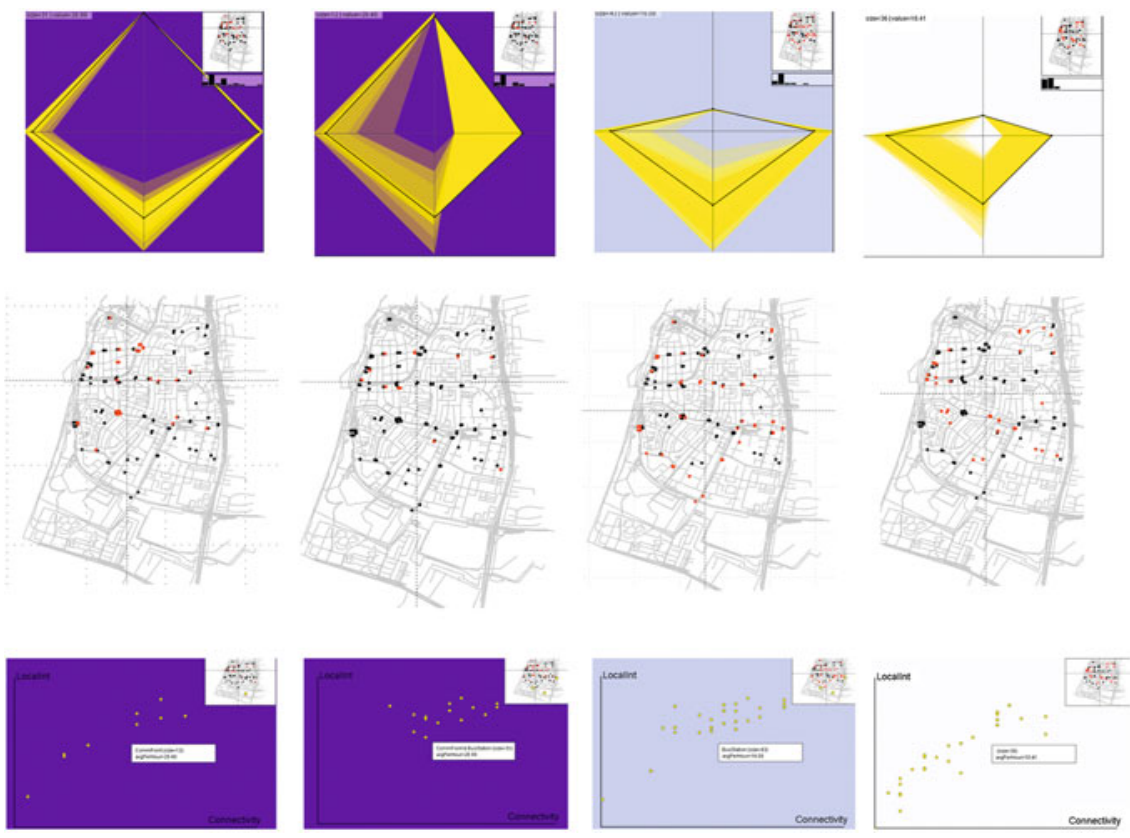

Fig. 2 Visual analysis of the Bat Yam Data. The top row shows the four different configurations of the functional-topological variables as found by cluster analysis for Bat Yam. The distribution of the measurements represented by the clusters on the map of Bat Yam in shown in the middle row. The bottom row shows scatter plots of the independent variables Connectivity (x-axis) versus LocalInt (y-axis) for the occurrences of CommFronts (first chart), CommFronts + BusStation (second chart), only BusStation (third chart), and neither presence of CommFronts or BusStations (fourth chart)

walkable configurations (with high values of pedestrians all the independent variables; left two clusters in Fig. 2, top row) are located mainly in the northeast part of the city. The other two configurations are distributed equally over the city areas. This unequal geographic distribution may be related to the central role of the northeast part of the city in its functioning and to a high level of connectivity and integration of its street network. We should notice in this respect that significance correlation was not found between the volume of pedestrian movement, and residential density or population size in different city areas. Therefore, we assume that part of the northeast area pedestrians come from other city areas due to its attractiveness. However, the focus of the investigation is on the distribution of pedestrians within a given area according to the selected functional and spatial variables.

To explore the exact relations among the values of selected variables in our data set, we also extended the visualization tool with scatter plot diagrams (see Fig. 2, bottom row). To illustrate the potential contribution of our tool, we chose to examine the relation between the two topological centrality variablesLocalIntegration and Connectivity, for the four possible combinations of 
CommFront and BusStations being present or absent. Our question was: What is the relationship between the values of these variables in each of the identified configurations? Specifically, we are interested in how the topological centrality values at street segment level are associated with the distributions of functional land uses (in our case, CommFront and BusStations) and with pedestrian movement. The accompanied scatter plots help to explore the interrelation among the three components-functional properties, spatial properties (topological centrality), and pedestrian movement-in different geographical areas. In the case we present, we can see that the topological centrality values tend to correlate positively. Within this general tendency, the more walkable configurations (high volume of pedestrian movement, darker background color in the scatter plot charts) tend to be with higher values of local integration. On the contrary, the less walkable configurations (third and fourth chart in the row) tend to represent street segments with low local integration and connectivity values. These findings indicate that the topological centrality variables create the basic conditions for both, distributions of functional land uses and pedestrian movement.

Figure 3 enhances the geographic exploration in more detail. We used our tool to produce a diagram that presents the volume of pedestrian movement (via circle size) in each point/segment, together with an indication to which of the clusters they belong (via color mapping). This enables obtaining essential information at different geographic scales on how the principal functional-spatial configurations are distributed in the geographic space, how they relate geographically to one another, and how each of them is related to the geographical distribution of pedestrian movements. It can assist us, for example, to locate areas that consist of segments of different functional-spatial configurations and walkability levels. Namely, it enables simultaneously to identify geographical areas with differential walkability levels and to clarify some of the reasons for that differentiation through investigation of the obtained configurations of independent variables. E.g., as shown in Fig. 3, some places comprise points that have similar environmental configurations, but differ in their walkability levels. In such situation, one of the main tasks in pedestrian planning policy is to find barriers and spatial partitions that prevent pedestrian flow in a given urban environments [e.g., (Hillier 2002; Orellana and Wachowicz 2011; Torrens 2012; Zook et al. 2012)]. Thus, such empiric knowledge leads to better designs of pedestrian paths that widen the areas of pedestrian movement; for example, as part of renewal of commercial areas [e.g., see Zampieri et al. (2009)].

\subsection{Tel Aviv Analysis}

The case of Tel Aviv is similar to that of Bat Yam with regard to the general interdependency among the spatial and functional independent variables and their relationships, as configuration types, with the volumes of pedestrian movements. The four-dimensional glyphs (see Fig. 4, top row) tend to expand rather equally in 


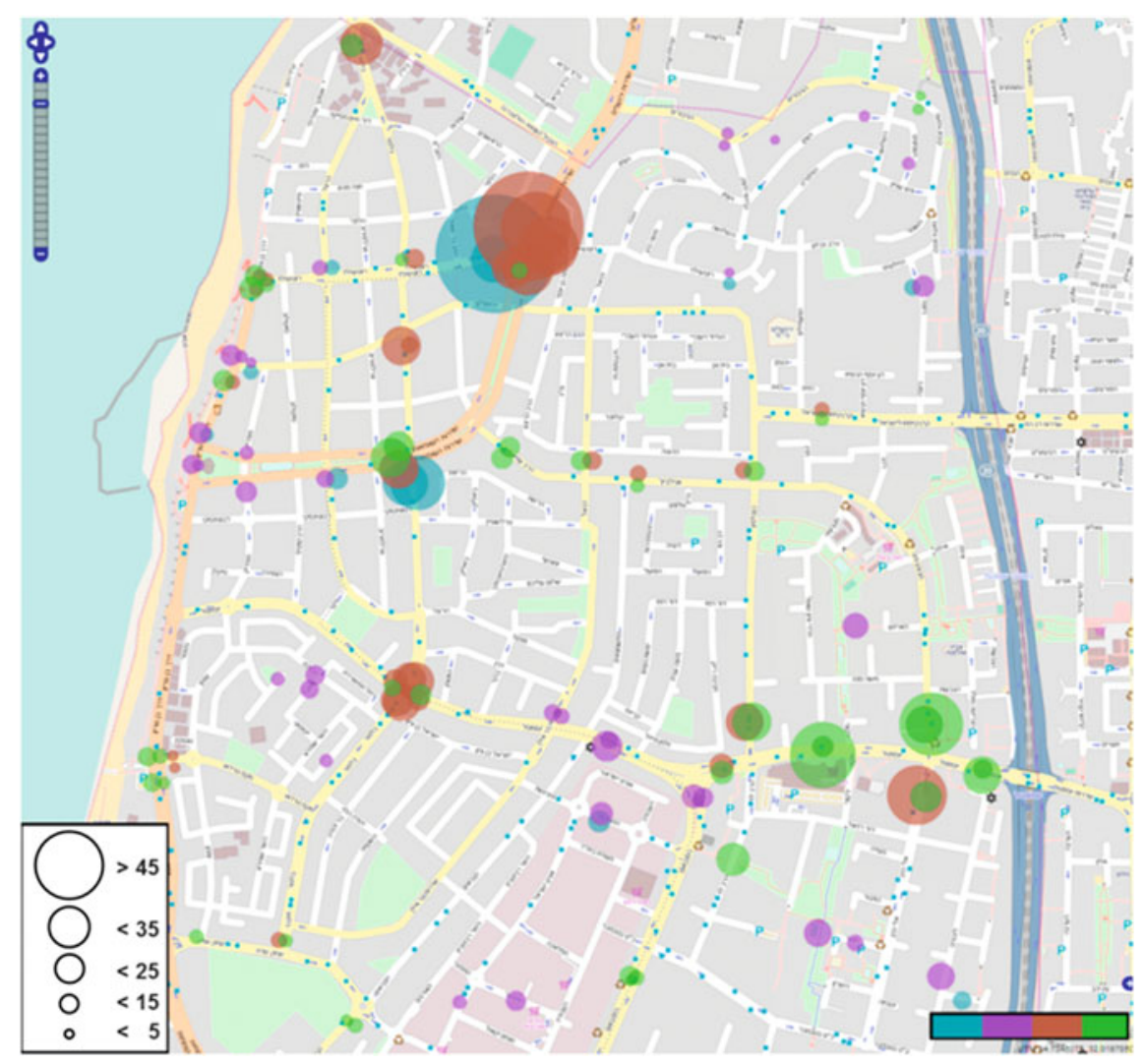

Fig. 3 The distribution of configuration types in selected street segments by volume of pedestrian movement for the city of Bat Yam. The colors (see legend in bottom-right part of image) denote the different configuration types from Fig. 2. Colors 1 and 3 correspond to configurations 1 and 2, and colors 2 and 4 to configurations 3 and 4

all directions, and along with that also varies the background color. This similarity between Tel Aviv and Bat Yam means interdependency among the urban environments' properties and positive correlation with the pedestrian movements. However, several significant differences exist between these two cases. First, while in Bat Yam, high values of independent variables are sufficient but not necessary conditions for creating significantly walkable locations, in Tel Aviv, high values of independent variables are sufficient as well as necessary for achieving such high walkability levels (see Fig. 4, the first cluster from left (note: Please zoom in for better readability). E.g., significant presence of pedestrians in Tel Aviv exists only where bus stops are located, and at the same time, bus stops are located in places where a significant presence of pedestrians exists. This indicates that there is stronger reciprocity between the urban environments' attributes and pedestrian movement. Second, a stronger interdependency and better fit also exists among the 

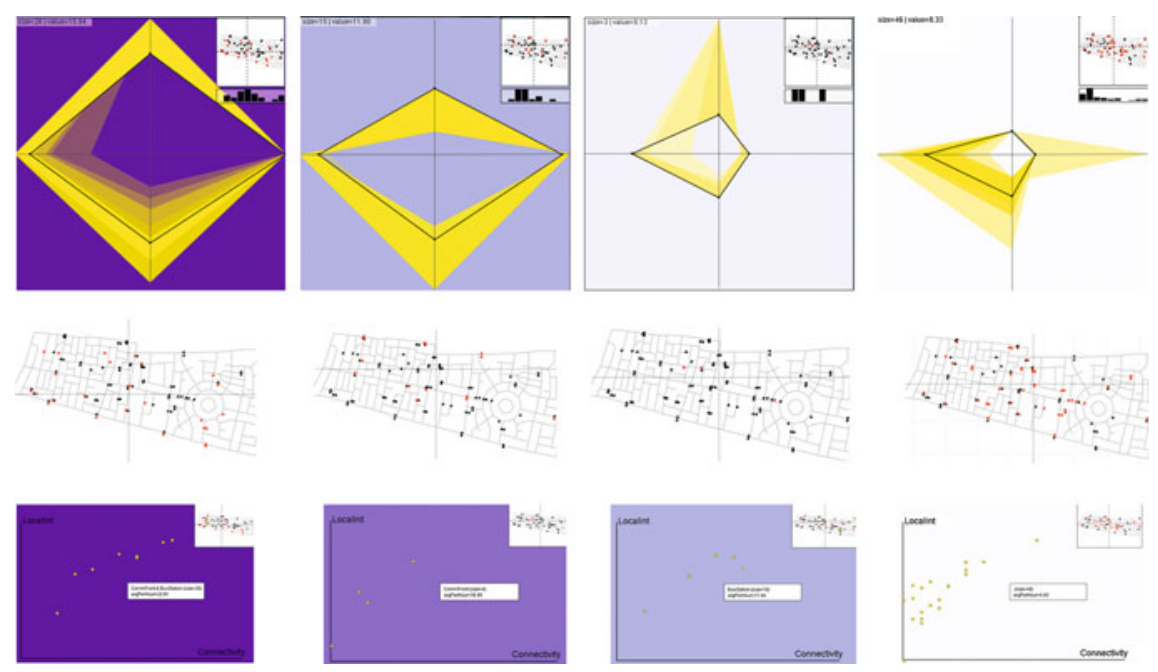

Fig. 4 Visual results for the Tel Aviv data set

independent variables themselves, especially between the two spatial variables (see the scatter plots of the independent variables in Fig. 4, bottom row). Thus, the results also indicate that more interdependency and even a structuration among all the variables involved in the phenomenon of urban pedestrian movement exists in Tel Aviv, which is more pronounced than in Bat Yam.

Third, the case of Tel Aviv differs greatly from the case of Bat Yam concerning the geographic distributions of the spatial-functional configurations. As Fig. 5 shows, in Tel Aviv the different configurations of variables are distributed equally and create a sort of hierarchical structure. We can see that the most walkable configuration type (denoted by the brown color), which tends to be with the higher volume (big circles), is distributed over all the area and creates a sort of skeleton or backbone, mainly in the western section, which was built earlier and is characterized by a street grid. At the same time, the other configuration types, with a relatively low volume of pedestrians, tend also to be located between and around the most walkable configurations, i.e., the locations with higher pedestrian movements. On the contrary, the different types of configurations in Bat Yam tend to concentrate in a few geographical areas with no clear spatial relation among them. We can also see that the locations with higher volume of pedestrian movement are located in two or three geographic areas that function only as "main spots" of pedestrian movements (see Fig. 5). That is, unlike Tel Aviv, Bat Yam is characterized by a geographical clustering of configuration types, and geographical inequality in the distribution of pedestrian movement. This can be seen as an expression of different spatial autocorrelations of the independent environmental variables. In Tel Aviv, the geographical scale of the land use mix is significantly more local, i.e., different land uses such as commerce and residence can be found in a relatively small geographical area. Accordingly, Tel Aviv also has much more 


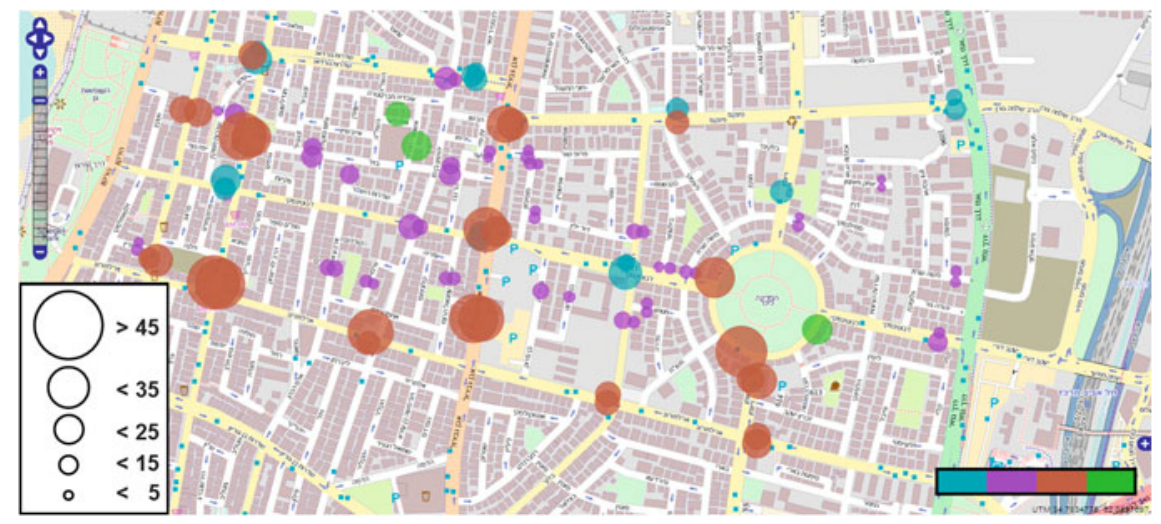

Fig. 5 Configuration types by volume of pedestrian movement for the city of Tel Aviv. The colors (see legend in bottom-right part of image) denote the different configuration types from Fig. 4. Colors 1 and 3 correspond to configurations 1 and 2, and colors 2 and 4 to configurations 3 and 4

equality in the geographic distribution of the volume of pedestrians at the level of individual locations and at the level of geographical areas. And again, much more correspondence exists between the configuration types and the volume of pedestrian movement.

\section{Discussion and Conclusion}

In this chapter, we explained and compared pedestrian movement patterns in two urban areas in different parts of the Tel Aviv metropolitan region. The first area, inner city Tel Aviv, referred to as Tel Aviv in this chapter, is the functional center of the metropolitan region that serves many non-local residents from the entire region. The second area is Bat Yam, a smaller city in the Tel Aviv metropolitan region, which serves mostly local residents.

Using adapted methods from visual data analysis, we found that these two urban areas differ greatly in several respects. Tel Aviv has much more correspondence and interdependency among the urban environments' variables. This is seen between the spatial and functional variables involved in the phenomenon of urban pedestrian movement. In addition, we found that this correspondence also has a geographical expression-pedestrian movements as well as configurations of independent environmental variables are distributed equally over the environment area and create a sort of hierarchical structure. On the contrary, Bat Yam has relatively less correspondence among the variables involved in the phenomenon of pedestrian movement and no significant spatial organization of pedestrian movements. These differences can be related mainly to the functional statuses of Bat Yam and Tel Aviv. While Bat Yam serves mostly local residents, Tel Aviv, as one 
of the main central areas of the metropolitan region, serves a relatively high rate of non-local residents, who are highly dependent on the functional activity in the area. In our case, this is commerce and bus stations. The findings we obtained by using visual analysis methods can assist policy making with the aim of improving the conditions for higher levels of walkability in urban environments throughout the metropolitan region.

Our approach is based on a visual, multidimensional correlation analysis. As such, it presents patterns of co-located measurements, and concentrates mainly on geometric properties. The results are determined by the selection of variables and the interactive analysis process. The study could be extended by considering additional descriptive factors, such as affordances of places like presence of tourist attractions, work place density, aesthetic criteria of the given architecture, and many more. Such factors could help to substantiate our initial findings. We already extend beyond the basic space syntax paradigm by including the functional variables CommFront and BusStation, but certainly, and extension would be interesting.

We identified a number of interesting items for future research. First, the geovisual analytics methods we developed for pedestrian movement analysis in different urban environments should be adapted for use by urban planners during the design of walkable environments, or when implementing walkability-oriented spatial policy. Second, the study could be extended to other urban parts in the metropolitan region to explore how pedestrian movements are created in different functional and morphological environments. Third, while we used the number of pedestrians as the empirical expression of pedestrian friendliness, qualitative analysis, e.g., by interviews, could further specify the exact notion of pedestrian friendliness. In addition, with the wide-spread use of smartphone technology, including wireless Internet and GPS modalities, we envision much more detailed and high-resolution pedestrian data becoming available in the future. Thereby, more complex patterns of pedestrian movements could be taken into account. Then, further advanced approaches for geovisual analytics need to be developed to adequately represent such extended measurements in user-friendly interfaces for use by planners and designers of walkable urban environments.

Acknowledgments We thank Sebastian Bremm and Tatiana von Landesberger of TU Darmstadt for fruitful discussions on the topic as part of a working group meeting of the DFG SPP 1335 on Scalable Visual Analytics.

\section{References}

Andrienko N, Andrienko G (2006) Exploratory analysis of spatial and temporal data-a systematic approach. Springer, Berlin

Bak P, Omer I, Schreck T (2010) Visual analytics of urban environments using high-resolution geographic data. Geospatial Thinking 25-42

Brewer C (2012) Color brewer 2.0 
Desyllas J, Duxbury E (2000) Planning for movement-measuring and modelling pedestrian flows in cities. In: Proceedings of the royal institute of chartered surveyors conference

Desyllas J, Duxbury E, Ward J, Hudson-Smith A (2003) Pedestrian demand modelling of large cities: an applied example from London. CASA working papers

Golledge R, Stimson R (1997) Spatial behavior: a geographic perspective. Guilford Press, New York

Guo D, Gahegan M, MacEachren A, Zhou B (2005) Multivariate analysis and geovisualization with an integrated geographic knowledge discovery approach. Cartography Geogr Inf Sci 32(2): 113

Hillier B (1996) Space is the machine. Cambridge University Press, Cambridge

Hillier B (2002) A theory of the city as object—or, how spatial laws mediate the social construction of urban space. Urban Des Int 7(3):153-179

Hillier B, Penn A, Hansonand J, Grajewski T, Xu J (1993) Natural movement: or, configuration and attraction in urban pedestrian movement. Environ Plann B: Plann Des 20(1):29-66

Inselberg A (2002) A survey of parallel coordinates. Math Vis 167-179

Jiang B (2007) A topological pattern of urban street networks: Universality and peculiarity. Physica A 384(2):647-655

Jiang B, Liu X (2010) Automatic generation of the axial lines of urban environments to capture what we perceive. Int J Geogr Inf Sci 24(4):545-558

Jolliffe I (2002) Principal component analysis. Springer, New York

Keim D, Kohlhammer J, Ellis G, Mansmann F (2011) Mastering the information age: Solving problems with visual analytics. Eurographics Assoc

Kohonen T (2001) Self-organizing maps. Springer, Berlin

Orellana D, Wachowicz M (2011) Exploring patterns of movement suspension in pedestrian mobility. Geogr Anal 43(3):241-260

Ozbil A, Peponis J, Stone B (2011) Understanding the link between street connectivity, land use and pedestrian flows. Urban Des Int 125-141

Ozer O, Kubat A (2007) Walking initiatives: a quantitative movement analysis. 6th international space syntax symposium

Porta P, Crucitti P, Latora V (2006) The network analysis of urban streets: a primal approach. Environ Plann B: Plann Des 5(33):705-725

Raford N, Ragland D (2006) Pedestrian volume modeling for traffic safety and exposure analysis: case of Boston, Massachusetts. Transportation research board 85th annual meeting

Spielman S, Thill J (2008) Social area analysis, data mining, and gis. Comput Environ Urban Syst 32(2):110-122

Thomas J, Cook K (2005) Illuminating the path: the research and development agenda for visual analytics. National visualization and analytics Ctr

Torrens Paul M (2012) Moving agent pedestrians through space and time. Ann Assoc Am Geogr 102:35-66

Turner A (2004) Depthmap 4-a researcher's handbook. School of Graduate Studies, UCL, Bartlett

Zampieri F, Rigatti D, Ugalde C (2009) Evaluated model of pedestrian movement based on space syntax, performance measures and artificial neural nets. In: Proceedings of the 7th international space syntax symposium, pp 1-8

Zhang L, Zuhang Y, Dai X (2012) A configuration study of pedestrian flows in multi-level commercial space. In: Proceedings of the 8th international space syntax symposium

Zook J, Lu Y, Glanz K, Zimring C (2012) Design and pedestrianism in a smart growth development. Environ Behav 44(2):216-234 